\title{
The effect of type I migration on the formation of terrestrial planets in hot-Jupiter systems
}

\author{
M. J. Fogg and R. P. Nelson
}

Astronomy Unit, Queen Mary, University of London, Mile End Road, London E1 4NS, UK

e-mail: [M.J.Fogg; R.P.Nelson]@qmul .ac.uk

Received 25 May 2007 / Accepted 16 July 2007

\begin{abstract}
Context. Our previous models of a giant planet migrating through an inner protoplanet/planetesimal disk find that the giant shepherds a portion of the material it encounters into interior orbits, whilst scattering the rest into external orbits. Scattering tends to dominate, leaving behind abundant material that can accrete into terrestrial planets.

Aims. We add to the possible realism of our model by simulating type I migration forces which cause an inward drift, and strong eccentricity and inclination damping of protoplanetary bodies. This extra dissipation might be expected to enhance shepherding at the expense of scattering, possibly modifying our previous conclusions.

Methods. We employ an $N$-body code that is linked to a viscous gas disk algorithm capable of simulating: gas accretion onto the central star; gap formation in the vicinity of the giant planet; type II migration of the giant planet; type I migration of protoplanets; and the effect of gas drag on planetesimals. We use the code to re-run three scenarios from a previous work where type I migration was not included.

Results. The additional dissipation introduced by type I migration enhances the inward shepherding of material but does not severely reduce scattering. We find that $>50 \%$ of the solids disk material still survives the migration in scattered exterior orbits: most of it well placed to complete terrestrial planet formation at $<3 \mathrm{AU}$. The shepherded portion of the disk accretes into hot-Earths, which survive in interior orbits for the duration of our simulations.

Conclusions. Water-rich terrestrial planets can form in the habitable zones of hot-Jupiter systems and hot-Earths and hot-Neptunes may also be present. These systems should be targets of future planet search missions.
\end{abstract}

Key words. planets and satellites: formation - methods: $N$-body simulations - astrobiology

\section{Introduction}

The core accretion hypothesis of giant planet formation postulates that giant planets originate in a cool location in a protoplanetary disk, beyond the snowline where the condensation of ices boosts the mass of solid material present (e.g. Pollack et al. 1996; Papaloizou \& Nelson 2005). This, and the fact that remoteness from the central star enhances the gravitational reach of accretion, may allow a $\sim 10 M_{\oplus}$ core to grow and then accumulate a massive gaseous envelope before the loss of the nebular gas which is observed to occur $\sim 1-10$ Myr after star formation (Haisch et al. 2001). The alternative gravitational instability model also predicts that giant planets will form in the cool outer disk regions (Boss 2000). Giant planets are also expected to migrate inward from their original formation location due to gravitational interactions with the disk, either during the core stage via type I migration, where the body remains embedded in the gas (e.g. Ward 1997; Papaloizou \& Larwood 2000; Tanaka et al. 2002; Tanaka \& Ward 2004), or via type II migration when the planet has grown massive enough $\left(\gtrsim 100 M_{\oplus}\right)$ to open a gap in the gas and becomes coupled to the inward viscous evolution of the disk (e.g. Lin \& Papaloizou 1986; Lin et al. 1996; Ward 1997; Nelson et al. 2000). According to recent models of the late stages of solar system formation, which appear to explain the current architecture of the solar system (Tsiganis et al. 2005; Morbidelli et al. 2005; Gomes et al. 2005), Jupiter and Saturn appear to have undergone only limited migration, suggesting that they may have formed quite late with respect to dispersal of the gas. Their influence on interior terrestrial planet formation at distances $\lesssim 2$ AU was probably modest, although recent models suggest secular interactions may have played an important role during gas disk dispersal (Nagasawa et al. 2005). In contrast, many giant exoplanets show evidence for substantial migration, being found well within what would have been their parent nebula's snowline (Butler et al. 2006), the most extreme examples being known as hot-Jupiters with examples found at distances $\lessgtr 0.1 \mathrm{AU}$ down to just a few stellar radii. These planets must have passed through their inner systems at an early time $(\sim 1-10 \mathrm{Myr})$ and would inevitably have had a strong influence on the formation of rocky planets that would otherwise be expected to grow there on timescales of 10-100 Myr (Chambers 2001; Kleine et al. 2002; Halliday 2004; O’Brien et al. 2006; Nimmo \& Kleine 2007).

It is expected that terrestrial planets external to a hot-Jupiter would have stable orbits (Jones et al. 2005) and should be able to form from any available pre-planetary material with a period ratio $\gtrsim 3$ (Raymond et al. 2005a). However, until recently it has been a common approach in both astrophysical and astrobiological literature to assume that terrestrial planets could not have formed in hot-Jupiter systems due to the disruptive effect of the giant planet's migration which is deemed to have cleared the inner system of planet-forming material (e.g. Ward \& Brownlee 2000; Lineweaver 2001; Armitage 2003; Lineweaver et al. 2004). However, recent work by two groups who have 
modeled terrestrial planet formation in the presence of type II giant planet migration have shown that this assumed clearing does not happen (Fogg \& Nelson 2005, 2006; Raymond et al. 2006; Fogg \& Nelson 2007; Mandell et al. 2007). Their simulations show that the giant planet shepherds the solids disk inward, compacting it and exciting the orbits of objects caught at first order resonances. Much of this excited material eventually experiences a close encounter with the giant planet and is expelled into an exterior orbit. The net effect of the giant's passage is therefore not the destruction and disappearance of the inner system disk, but instead a modest dilution and strong excitation and radial mixing of solid material. Over the entire course of the migration, a new disk of solid material builds up in orbits external to the final position of the hot-Jupiter where terrestrial planet formation can resume. The results of further simulation of accretion in this scattered disk suggest that terrestrial planets should be commonplace in hot-Jupiter systems: worlds that might possibly be water-rich due to strong inward mixing of material from beyond the nebular snowline (Raymond et al. 2006; Fogg \& Nelson 2007; Mandell et al. 2007).

All these papers agree that the outcome of giant planet migration through an inner system solids disk is to partition most of its mass into the exterior scattered remnant described above and an interior remnant resulting from shepherded material that is not scattered by the giant. This shepherded fraction is compacted into the restricted volume close to the central star, where dynamical times are short, resulting in a rapid burst of accretion with the potential to form stable hot-Neptune or hot-Earth type planets orbiting interior to the final position of the giant (see also Zhou et al. 2005). Comparing results however reveals that the relative predominance of scattering versus shepherding is significantly influenced by the strength of dissipative forces (gas drag and dynamical friction) operating in each particular model. Whilst interior and exterior remnants are found in all cases, a distinct trend is evident favoring shepherding under conditions of strong dissipation and scattering when dissipation is weaker. For example, the model in Fogg \& Nelson (2005) assumed a steady-state gas disk with a fixed $r^{-1.5}$ surface density profile which maximized both the influence of gas drag on planetesimal sized objects and hence their ability to damp the orbits of protoplanets via dynamical friction. The formation of shepherded hot-Neptunes and hot-Earths was a common outcome of these simulations with as little as $\sim 26 \%$ of the original disk material being scattered into the external disk in the youngest scenarios. (In the case of constant gas density the average strength of dynamical friction still falls with disk maturity as planetesimals are cleared through accretion.) In contrast, in their latest model (Fogg \& Nelson 2007) which incorporates a time dependent, viscously evolving, gas disk with a partial central cavity and annular gap centered on the giant planet's orbit, dissipation is much lower and declines with time, especially in the inner regions of the system. In this perhaps more realistic case, hot-Earths start to form in the shepherded portion of the disk but are almost always scattered or accreted by the giant at late times when it has migrated down to $a \lesssim 0.5 \mathrm{AU}$. Irrespective of the maturity of the solids disk at the start of migration, $>60 \%$ of its mass is always scattered into an external disk. Dissipation levels within the model of Raymond et al. (2006) and Mandell et al. (2007), with its linearly declining, fixed surface density profile gas disk, appear to lie intermediate between the two models of Fogg \& Nelson. Shepherded hot-Earths sometimes survive at the end of their simulations and sometimes not, although a rigorous comparison between their work and that of Fogg \& Nelson is not possible due to differences in assumed initial conditions, and the fact that in most of their simulations the giant planet comes to rest at a radial distance of $>0.1 \mathrm{AU}$.

One potentially important physical process neglected in these models is type I migration which operates on sub-gap opening bodies of $\sim 0.1-100 M_{\oplus}$ (e.g. Ward 1997; Papaloizou \& Larwood 2000; Tanaka et al. 2002; Tanaka \& Ward 2004). Type I migration is generated by the asymmetric torques exerted on a protoplanet from the wakes it creates in the gas disk and is expected to impart an inward radial drift and strong eccentricity and inclination damping that increases in effectiveness linearly with protoplanetary mass. The realism of type I migration has long been controversial as theoretically predicted spiral-in times can be so short as to threaten protoplanetary survival and to render an explanation of the solar system's architecture particularly difficult. Many planet formation models therefore ignore this process entirely. It might be that type I migration does not operate as rapidly as predicted: one proposal being that stochastic torques from density fluctuations in a turbulent disk could superimpose a random walk in semi-major axis over the smooth inward drift predicted by theory (Nelson \& Papaloizou 2004; Laughlin et al. 2004; Nelson 2005). However, it is becoming apparent that even near-nominal rates of type I migration are not necessarily fatal to planet formation and survival. Work by McNeil et al. (2005) has shown that it is possible to grow a terrestrial planetary system in a simulation including type I migration forces by enhancing the original quantity of solid material present whilst including a rapidly dissipating gas disk. The model of Daisaka et al. (2006) succeeds in retaining sufficient material in the terrestrial region by invoking a shorter gas depletion timescale in the inner system than the observational value (based on infrared observations at $a \sim 100 \mathrm{AU}$ ), or if the the initial gas to dust ratio is smaller than the conventional minimum mass solar nebula (MMSN) model. The problem with preventing the more massive giant planet cores from being lost to the central star is of greater severity, but recent work by Thommes \& Murray (2006) suggests that this may be possible late in the lifetime of the gas disk when accretion and migration timescales become comparable. Proposed system properties that might widen such a window for core survival include small planetesimal sizes, low midplane gas disk viscosities and enhanced collision cross sections due to core atmospheres (Chambers 2006a; Thommes et al. 2007).

The possible realism of strong type I migration forces is of obvious and perhaps critical relevance to the above-cited models of giant planet migration through an inner system solids disk. Even if the issue of pre-existing type I migration of the giant planet's core is neglected and the assumption of the fully formed giant planet undergoing type II migration is retained, type I migration forces would still be expected to operate on the protoplanetary components of the inner system disk, introducing another source of damping additional to dynamical friction. Extra dissipation would be expected to affect the partitioning of inner system material by enhancing the shepherded fraction at the expense of the scattered fraction, potentially invalidating all the previous claims that sufficient solid material survives the passage of the giant to permit the formation of terrestrial planets. Our primary motivation is to investigate this issue here by adding type I migration forces to the model of Fogg \& Nelson (2007) and re-running one early and two late scenarios from that paper. We note that additional sources of damping may also be important such as damping by collisional debris, but inclusion of these effects is beyond the scope of our model. In Sect. 2 we outline the additions to our model and the initial conditions of the simulations; in Sect. 3 the results are presented, discussed, and 
compared to previous work; in Sect. 4 we consider some caveats, and in Sect. 5 we offer our conclusions.

\section{Description of the model}

We model planetary accretion using a modified version of the Mercury 6 hybrid-symplectic integrator (Chambers 1999), run as an $N+N^{\prime}$ simulation, where we have $N$ protoplanets embedded in a disk of $N^{\prime}$ "super-planetesimals" - particles with masses a tenth that of the initial masses of protoplanets which act as statistical tracers of a much larger number of real planetesimals and are capable of exerting dynamical friction on larger bodies (e.g. Thommes et al. 2003). The protoplanets (and the giant when it is introduced) interact gravitationally and can accrete and merge inelastically with all the other bodies in the simulation, whereas the super-planetesimal population is non-self-interacting. Super-planetesimals however are subject to a drag force from their motion relative to the nebular gas that is equivalent in its dynamical action to the gas drag that would be experienced by a single $10 \mathrm{~km}$ radius planetesimal. A detailed outline of these aspects of our model is given in Fogg \& Nelson (2005).

We model the evolution of the nebular gas using a $1 \mathrm{D}$ viscous disk model that is linked to the $N$-body code with a timestep synchronization routine. This model solves numerically a viscous gas disk diffusion equation that is modified to include the tidal torques exerted by an embedded giant planet (Lin \& Papaloizou 1986; Takeuchi et al. 1996) and its implementation is fully described in Fogg \& Nelson (2007). The gas responds under the influence of this algorithm by depleting over time via viscous accretion onto the central star; by opening up an annular gap centered on the giant planet's orbit; and by forming a partial inner cavity due to dissipation of propagating spiral waves excited by the giant planet. The back reaction of these effects on the giant planet is resolved as torques which drive type II migration in a fully self-consistent manner.

\subsection{Type I migration forces}

We have added type I migration forces to our model, which are applied to protoplanets only, by implementing the approach of Cresswell \& Nelson (2006) who adopted the migration time prescription of Tanaka et al. (2002) and eccentricity damping time prescription of Tanaka \& Ward (2004) and modified them with factors derived by Papaloizou \& Larwood (2000) to describe evolution in the case of large eccentricity.

Their formula for the type I migration time is:

$t_{\mathrm{m}}=\frac{2}{2.7+1.1 \beta}\left(\frac{M_{*}}{m}\right)\left(\frac{M_{*}}{\Sigma_{\mathrm{g}} a^{2}}\right)\left(\frac{h}{r}\right)^{2}\left(\frac{1+\left(\frac{e r}{1.3 h}\right)^{5}}{1-\left(\frac{e r}{1.1 h}\right)^{4}}\right) \Omega^{-1}$,

where $M_{*}$ is the mass of the central star, $\Sigma_{\mathrm{g}}$ is the gas surface density in the vicinity of the planet, $h$ is the gas scale height, and $m, a, r, e$ and $\Omega$ are the planet's mass, semi-major axis, distance from the star, orbital eccentricity and orbital frequency respectively. The factor $\beta$ in the first term is the gas disk surface density profile index $\left(\Sigma_{\mathrm{g}} \propto r^{-\beta}\right)$, which we take to be fixed at its initial value of $\beta=1.5$, even though $\beta$ falls in value during the evolution of the inner disk as gas drains onto the star. In practise, this simplification makes only a $\sim 15 \%$ difference to $t_{\mathrm{m}}$ at later times and avoids the need for additional, time-consuming, measurement of $\beta$ at every protoplanetary location each gas disk time step. Important behavioral features to note from Eq. (1) are that type I migration speeds up with an increase in planetary mass and slows down with a decrease in gas density or increased eccentricity. When $e>1.1 \mathrm{~h} / \mathrm{r}$, inward migration halts as $t_{\mathrm{m}}$ becomes negative and only resumes when eccentricity is damped to lower values. We note that under certain circumstances, such as there being a surface density jump, or an optically thick disk, or MHD turbulence, type I migration may be substantially modified or reversed (Masset et al. 2006; Paardekooper \& Mellema 2006; Papaloizou \& Nelson 2005; Nelson 2005). Consideration of these effects goes beyond the scope of this paper.

The control of $\Sigma_{\mathrm{g}}(r, t)$ by our viscous disk algorithm causes $t_{\mathrm{m}}(r, t)$ to behave in an interesting way. Since $t_{\mathrm{m}} \propto$ $\Sigma_{\mathrm{g}}^{-1} a^{-2}(h / r)^{2} \Omega^{-1}$ and $\Sigma_{\mathrm{g}} \propto r^{-\beta}$, and as we use $h / r=$ $0.047(r / \mathrm{AU})^{1 / 4} \mathrm{AU}$, we find $t_{\mathrm{m}} \propto r^{\beta}$ and $\dot{r} \propto r^{1-\beta}$. At early times and in our outer disk, $\beta \simeq 1.5$ and $\dot{r} \propto r^{-1 / 2}$, so migration is faster for interior objects of a given mass. However, the viscous draining of gas onto the central star rapidly results in much of the nebula declining to a shallower profile index of $\beta \simeq 1$, falling further to $\beta \simeq 0.75$ interior to $1 \mathrm{AU}$ (see Fig. 1 in Sect. 2.2); in this situation $\dot{r} \propto r^{1 / 4}$, so relative migration rates are slower for interior objects.

Cresswell and Nelson's formula for eccentricity damping time is:

$t_{\mathrm{e}}=\frac{Q_{\mathrm{e}}}{0.78}\left(\frac{M_{*}}{m}\right)\left(\frac{M_{*}}{\Sigma_{\mathrm{g}} a^{2}}\right)\left(\frac{h}{r}\right)^{4}\left(1+\frac{1}{4}\left(e \frac{r}{h}\right)^{3}\right) \Omega^{-1}$,

where $Q_{\mathrm{e}}=0.1$ was chosen as a normalization factor to get $t_{\mathrm{e}}$ into good agreement with values measured from hydrodynamic simulations. We additionally assume that the timescale for inclination damping is the same as that for eccentricity damping, i.e. $t_{\mathrm{i}}=t_{\mathrm{e}}$. As with the behavior for inward migration, eccentricity damping is also stronger with increasing planetary mass and weaker with decreasing gas density or higher eccentricity.

Given $t_{\mathrm{m}}$ and $t_{\mathrm{e}}$, type I migration forces are then applied via the following accelerations to each protoplanet:

$\boldsymbol{a}_{\mathrm{m}}=-\frac{\boldsymbol{v}}{t_{\mathrm{m}}}$

$\boldsymbol{a}_{\mathrm{d}}=-2 \frac{(\boldsymbol{v} \cdot \boldsymbol{r}) \boldsymbol{r}}{r^{2} t_{\mathrm{e}}}-2 \frac{(\boldsymbol{v} \cdot \boldsymbol{k}) \boldsymbol{k}}{t_{\mathrm{i}}}$

where $\boldsymbol{v}$ is the protoplanet's velocity vector and $\boldsymbol{k}$ is a unit vector in the vertical direction.

\subsection{Initial conditions and running of the simulations}

As an initial state, we assume the presence of a protoplanetary disk about a $1 M_{\odot}$ star conforming in properties and structure to the minimum mass solar nebula model of Hayashi (1981), but scaled up by a factor of three. We assign a nominal age of this disk to be $\sim 0.5 \mathrm{Myr}$ (this being the $t=0$ start time for the simulations) by which point the evolution of the solid components of the inner disk is considered to have reached the oligarchic growth stage described by Kokubo \& Ida (2000). This inner solids disk extends initially between $0.4-4.0 \mathrm{AU}$ and has a snowline at 2.7 AU beyond which its surface density is boosted by a factor of 4.2 to account for ice condensation. The generation of the $N$-body components of this initial solids disk assumes initial protoplanetary masses of 0.025 and $0.1 M_{\oplus}$ interior and exterior to the snowline respectively, spaced approximately 8 mutual Hill radii apart, with the remainder of the material inventory consisting of super-planetesimals with a fixed mass of $10 \%$ of the initial masses of the local protoplanets. Further details are described in Fogg \& Nelson (2005) and relevant data are shown in Table 1 
Table 1. Data describing initial solids disk set-up.

\begin{tabular}{c|cc|c}
\hline \hline & Rocky zone & Icy zone & Total \\
& $0.4-2.7 \mathrm{AU}$ & $2.7-4.0 \mathrm{AU}$ & $0.4-4.0 \mathrm{AU}$ \\
\hline$M_{\text {solid }}$ & $9.99 M_{\oplus}$ & $14.8 M_{\oplus}$ & $24.8 M_{\oplus}$ \\
\hline$m_{\text {proto }}$ & $0.025 M_{\oplus}$ & $0.1 M_{\oplus}$ & \\
$N$ & 66 & 9 & 75 \\
\hline$m_{\text {s-pl }}$ & $\begin{array}{c}0.0025 M_{\oplus} \\
N^{\prime}\end{array}$ & $\begin{array}{c}0.01 M_{\oplus} \\
1336\end{array}$ & 1392 \\
\hline$f_{\text {proto }}$ & 0.17 & 0.06 & 4278 \\
\hline
\end{tabular}

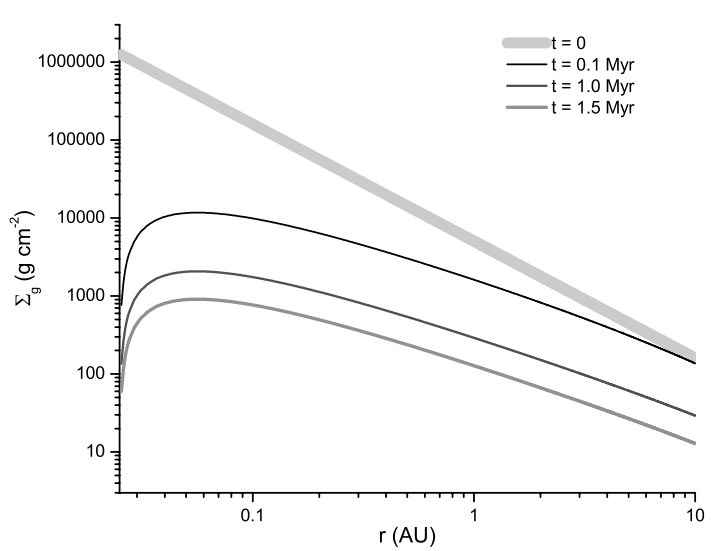

Fig. 1. Evolution of the gas surface density within the inner $10 \mathrm{AU}$ of our simulated disk. The upper line is the initial $r^{-1.5} \Sigma_{\mathrm{g}}$-profile for a $3 \times$ MMSN disk. The lower curves, in descending order, are the profiles at $0.1,1.0$ and $1.5 \mathrm{Myr}$ respectively.

which gives, for zones interior and exterior to the snowline, values for the total mass of solid material $M_{\text {solid }}$, the number and mass of protoplanets $N$ and $m_{\text {proto }}$, and the number and mass of super-planetesimals $N^{\prime}$ and $m_{\mathrm{s}-\mathrm{pl}}$. The parameter $f_{\text {proto }}$, at the foot of Table 1, is the mass fraction of the solids disk contained in protoplanets and we use this here as a rough measure of the evolution of the disk, taking $f_{\text {proto }}=0.5$ to denote the transition between oligarchic and giant impact growth regimes. We model the gas component of the disk from 0.025-33 AU with an initial surface density profile of $\Sigma_{\mathrm{g}} \propto r^{-1.5}$ which gives an initial mass of $0.0398 M_{\odot} \cong 42 M_{\mathrm{J}}$. The disk alpha viscosity is $\alpha=2 \times 10^{-3}$ which gives a viscous evolution time at $5 \mathrm{AU} \simeq 120000$ years and a mass depletion e-folding time $\simeq 580000$ years (see Fogg $\&$ Nelson 2007, for details).

From $t=0$ we start by running this combined $N$-body and viscous disk model in the absence of the giant planet in order to mature it to different ages. This is done with a symplectic time step of 8 days and a simulation inner edge of $0.1 \mathrm{AU}$, interior to which any solid material is eliminated. Our previous simulations have shown the importance of this maturation as the relative strengths of dissipative forces (gas drag, dynamical friction, and the newly included type I migration) all vary with time, as the gas density falls and protoplanets grow at the expense of planetesimals. We generate one "early" and two "late" scenarios by ageing the system for $0.1,1.0$ and $1.5 \mathrm{Myr}$ that are the equivalent of scenarios I, IV and V from Fogg \& Nelson (2007) and to facilitate comparison with this previous work we keep this nomenclature here.

The initial gas surface density and its profile at these three ages are plotted in Fig. 1 and numerical data for the evolved disks, including the remaining mass of gas $\left(M_{\text {gas }}\right)$ and solid material $\left(M_{\text {solid }}\right)$, the maximum protoplanetary mass $\left(m_{\max }\right)$, the
Table 2. Disk data: after 0.1, 1.0 and 1.5 Myr of evolution.

\begin{tabular}{c|ccc}
\hline \hline Time (Myr) & 0.1 & 1.0 & 1.5 \\
Scenario ID & $\mathrm{I}$ & $\mathrm{IV}$ & $\mathrm{V}$ \\
\hline$M_{\text {gas }}\left(M_{\mathrm{J}}\right)$ & 30 & 7 & 3 \\
$M_{\text {solid }}\left(M_{\oplus}\right)$ & 24.8 & 24.8 & 23.5 \\
$m_{\max }\left(M_{\oplus}\right)$ & 0.55 & 1.07 & 1.83 \\
$N$ & 51 & 24 & 16 \\
$N^{\prime}$ & 4087 & 2171 & 1585 \\
$f_{\text {proto }}$ & 0.20 & 0.47 & 0.51 \\
\hline
\end{tabular}

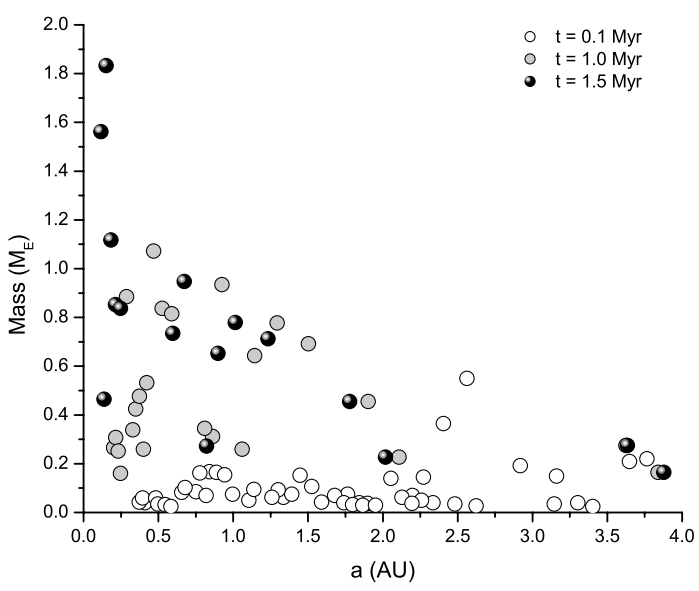

Fig. 2. Protoplanetary masses vs. semi-major axis obtained after evolution to the starting points of scenarios I, IV and V. Substantial inward drift is visible at late times due to type I migration.

number of surviving particles $\left(N \& N^{\prime}\right)$, and the protoplanet mass fraction $\left(f_{\text {proto }}\right)$ are given in Table 2 . Large reductions in gas density are illustrated, especially in the inner regions of the system. Particle numbers fall and $m_{\max }$ and $f_{\text {proto }}$ rise with time as expected and by the time of the latest scenario at $t=1.5 \mathrm{Myr}$, some solid material has been lost within 0.1 AU. It might be thought that this loss (just $\sim 5 \%$ of our solids inventory) is considerably less than would be expected given our inclusion of type I migration forces, however at early times the protoplanetary masses are too small to be greatly effected by inward type I drift and by the time they have grown an order of magnitude to $\sim M_{\oplus}$, inward drift remains slow as the gas density in the inner system has fallen by an even larger factor. The state of the solid components of the disk at the starting point of scenario I shows little difference to its equivalent case with no type I migration in Fogg \& Nelson (2007); however by the time the system is aged to the starting point of scenarios IV and V, substantial inward movement of the protoplanetary population has happened, depleting the outer disk and crowding the inner regions with protoplanets (see Fig. 2). The adequacy of an 8 day time-step for our late maturation runs is questionable as significant material has moved inside $0.4 \mathrm{AU}$ at $t>1 \mathrm{Myr}$. However, protoplanetary growth in the inner part of our model disk occurs rapidly and mostly at distances of $>0.4 \mathrm{AU}$ and tests we conducted to examine this issue showed that disk forces prevent strongly divergent behavior as a function of step size. The mass distribution of our matured disks interior to $0.4 \mathrm{AU}$ is therefore not significantly affected by our choice of step size and, once the giant planet is inserted at the start of each scenario, orbital evolution is resolved with a shorter time-step of one tenth the orbital period of the innermost object. As well as type I migration generating edge effects at the inner edge of our solids disk, it is likely that it would drive in other objects into our simulation region from 


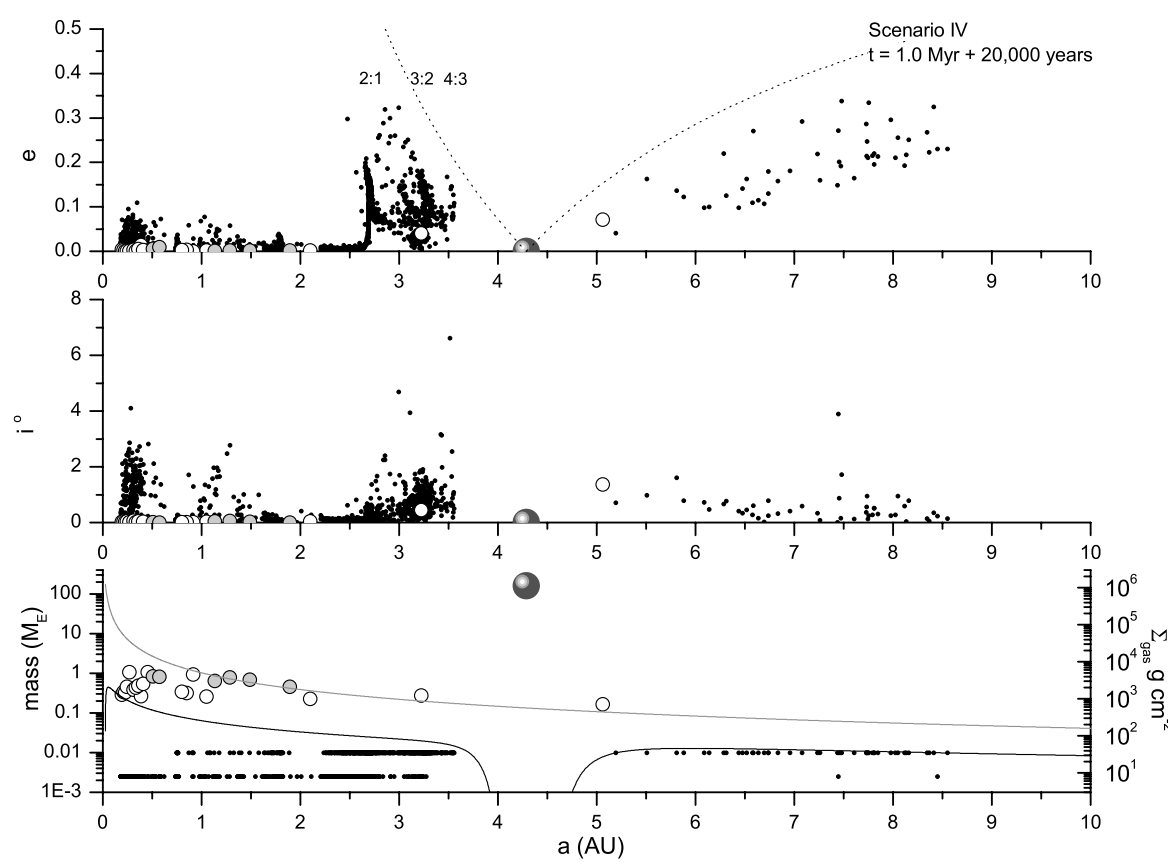

Fig. 3. Scenario IV at 20000 years after the start of giant planet migration, showing the mass, inclination and eccentricity of objects. Small black dots represent super-planetesimals; white filled circles are rocky protoplanets; grey filled circles are icy protoplanets and the large highlighted grey filled circle is the giant. Objects plotted between the dotted lines in the upper panel have orbits that intersect the orbit of the giant. The location of the 2:1, 3:2 and $4: 3$ resonances with the giant are indicated. Gas surface density is read on the right hand axis of the lower panel, the upper grey curve being the unevolved profile at $t=0$ and the lower black curve being the current profile. beyond 4 AU over this time: we have not included this possibility here but comment on it further in Sect. 4 .

The three aged disks summarized in Table 2 are used as the basis for the type II giant planet migration scenarios studied here. In each case, a giant planet of $0.5 M_{\mathrm{J}}$ is inserted at $5 \mathrm{AU}$ after removing $0.4 M_{\mathrm{J}}$ of gas from the disk between 3-7 AU and the inner boundary of the simulation is reset to $0.014 \mathrm{AU}$, which is approximately the radius of a $\mathrm{T}$ Tauri star (Bertout 1989). The giant planet then proceeds to clear an annular gap in the gas and migrates inward in step with its viscous evolution. The simulations are halted when the giant reaches hot-Jupiter territory at $0.1 \mathrm{AU}$ : in scenario I, this takes 114000 years, in close agreement with the viscous disk evolution time at $5 \mathrm{AU}$, but in scenarios IV and V the process takes longer ( $\sim 152500$ and 220000 years respectively) because at their late times of starting most of the gas has been lost (see Table 2) and the remainder is less effective at driving migration. The symplectic time-step for these runs was set to one tenth the orbital period of the innermost object which was achieved by dividing each simulation into a set of sequential sub-runs with the time-step adjusted appropriately at each re-start. At late times in each scenario when material had drifted interior to $0.1 \mathrm{AU}$, this entailed working with time-steps of less than one day with overall run times of several months as a consequence.

\section{Results}

We describe first the behavior of one of our runs in detail and then proceed to discuss the effect of adding type I migration to our model by contrasting the results of our three scenarios presented here with their counterparts in Fogg \& Nelson (2007).

\subsection{An account of scenario IV}

Scenario IV is one of our "late" scenarios, taking place $1 \mathrm{Myr}$ after our model start time at a system age of $\sim 1.5 \mathrm{Myr}$. By this point, and after removing the gas required for the giant planet's envelope, the amount of gas remaining in the disk has fallen to just $16 \%$ of its initial value with the result that the giant takes
152000 years to migrate inward to $0.1 \mathrm{AU}$, longer than would be predicted from the viscous evolution time. Four snapshots of the evolution of Scenario IV are illustrated in Figs. 3-6 showing the mass, inclination and eccentricity of objects, and the gas surface density vs. semi-major axis. The original provenance of the protoplanets (interior or exterior to the snowline) is denoted by the shading of its symbol as described in the caption to Fig. 3. In the case of a merger between rocky and icy protoplanets, this shading is determined by that of the most massive of the pair.

An early stage in the run at 20000 years after the introduction of the giant planet is shown in Fig. 3. The giant has moved in to $4.29 \mathrm{AU}$ and has caused significant excitation of the outer solids disk material at first order resonances. Noticeable also is that the outer disk beyond $\sim 2$ AU has been largely cleared of protoplanets due to previous type I migration. Only two low mass protoplanets remain in this region, one of them having recently become caught at the interior 3:2 resonance with the giant planet and the other having already been scattered into an external orbit.

An intermediate stage in scenario IV at 80000 years is shown in Fig. 4 where the giant planet has migrated inward to 2.04 AU and is at the point of entering the zone that is crowded with protoplanets at $a<2$ AU. Most of the planetesimals originating in the outer disk have now been shepherded interior to $2 \mathrm{AU}$, greatly increasing the surface density of solids caught between the 2:1 and 4:3 resonances. Two protoplanets have been captured at the 3:2 resonance and are in the process of having their orbits excited to an eccentricity where they will cross the giant planet's orbit and become vulnerable to scattering or accretion. Type I eccentricity damping is therefore not always strong enough to counteract the resonant pumping exerted by the giant planet as is shown by the additional $0.46 M_{\oplus}$ protoplanet that has been expelled into the growing scattered disk. One reason for this is that $t_{e}$ increases with the cube of eccentricity (Eq. (2)) greatly reducing the resistance of type I eccentricity damping to strong perturbations. It is also evident by this stage that the giant planet is easily capable of catching up and overtaking the protoplanets which have only moved inward slightly in the meantime. For example, the type I migration time given by Eq. (1) for a $0.5 M_{\oplus}$ protoplanet at $2 \mathrm{AU}$, with $e=0, h / r=0.056$ and 

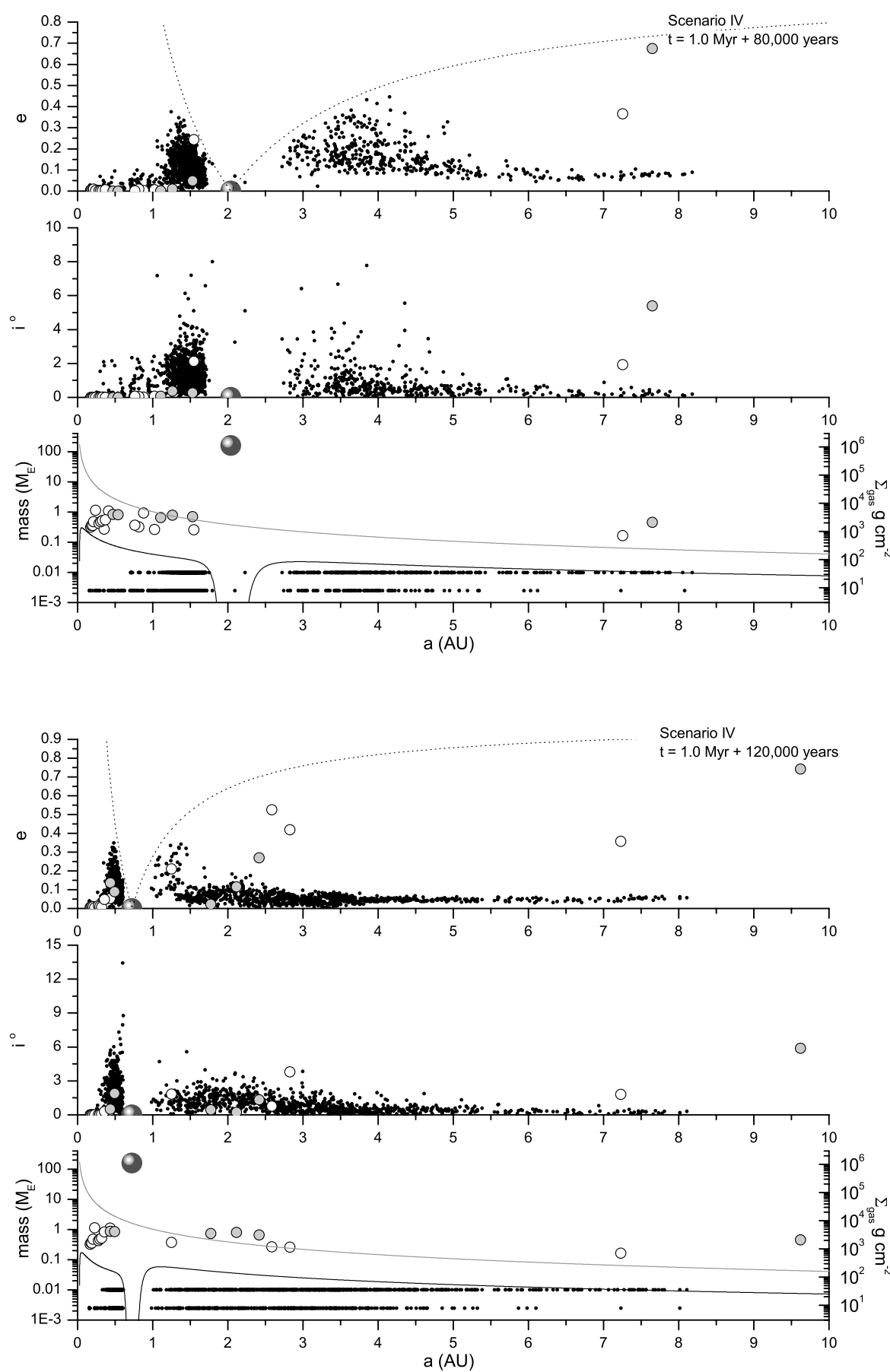

Fig. 4. Scenario IV at 80000 years after the start of giant planet migration. The giant has now moved inward to $2.04 \mathrm{AU}$ and is beginning to enter the inner zone that has become packed with protoplanets due to previous type I migration.
Fig. 5. Scenario IV at 120000 years after the start of giant planet migration. The giant has now moved inward to $0.72 \mathrm{AU}$. Six more protoplanets and a substantial number of planetesimals have been scattered into the external disk.
$\Sigma_{\mathrm{g}}=150 \mathrm{~g} \mathrm{~cm}^{-2}$ (a twelve-fold reduction in gas surface density at this location from that at $t=0$ ) is $t_{\mathrm{m}} \approx 6 \mathrm{Myr}$, comfortably larger than the type II migration time of the giant.

Figure 5 shows the system 120000 years after the introduction of the giant planet which has now reached 0.72 AU. An external disk is building up. Eight more protoplanets have had close encounters with the giant planet, resulting in two of them being accreted and six undergoing external scattering. The orbits of the most massive and least eccentric of these bodies are seen to be damping fast, as would be expected from the influence of Eq. (2). Some of this damping might also be due to dynamical friction as a substantial number of super-planetesimals have also been scattered into the same vicinity. Most of the solids mass however remains in the shepherded disk fraction, interior to the giant planet. Gas drag and type I damping forces in this region however are now two orders of magnitude weaker than a nominal $3 \times$ MMSN model as gas accretion onto the central star has reduced $\Sigma_{\mathrm{g}}$ here by a factor of $\sim 25$ since $t=0$ and dissipation of spiral waves generated by the approaching giant have recently reduced $\Sigma_{\mathrm{g}}$ by an additional factor of $\sim 4$.

Scenario IV ends at 152500 years after the introduction of the giant planet when it reaches $0.1 \mathrm{AU}$ and the system configuration at this point is shown in Fig. 6. The final 0.6 AU of the giant's migration has had a destructive effect on the densely packed inner system material. Of the twelve protoplanets remaining interior to the giant at the point shown in Fig. 5, there are only two remaining: a $0.79 M_{\oplus}$ planet scattered to $0.8 \mathrm{AU}$, and a $1.77 M_{\oplus}$ hot-Earth candidate at $0.062 \mathrm{AU}$, close to the $2: 1$ resonance, with an eccentric, inclined orbit $\left(e=0.46, i=25.3^{\circ}\right)$. The other ten, which were poorly damped due to low gas densities, and 


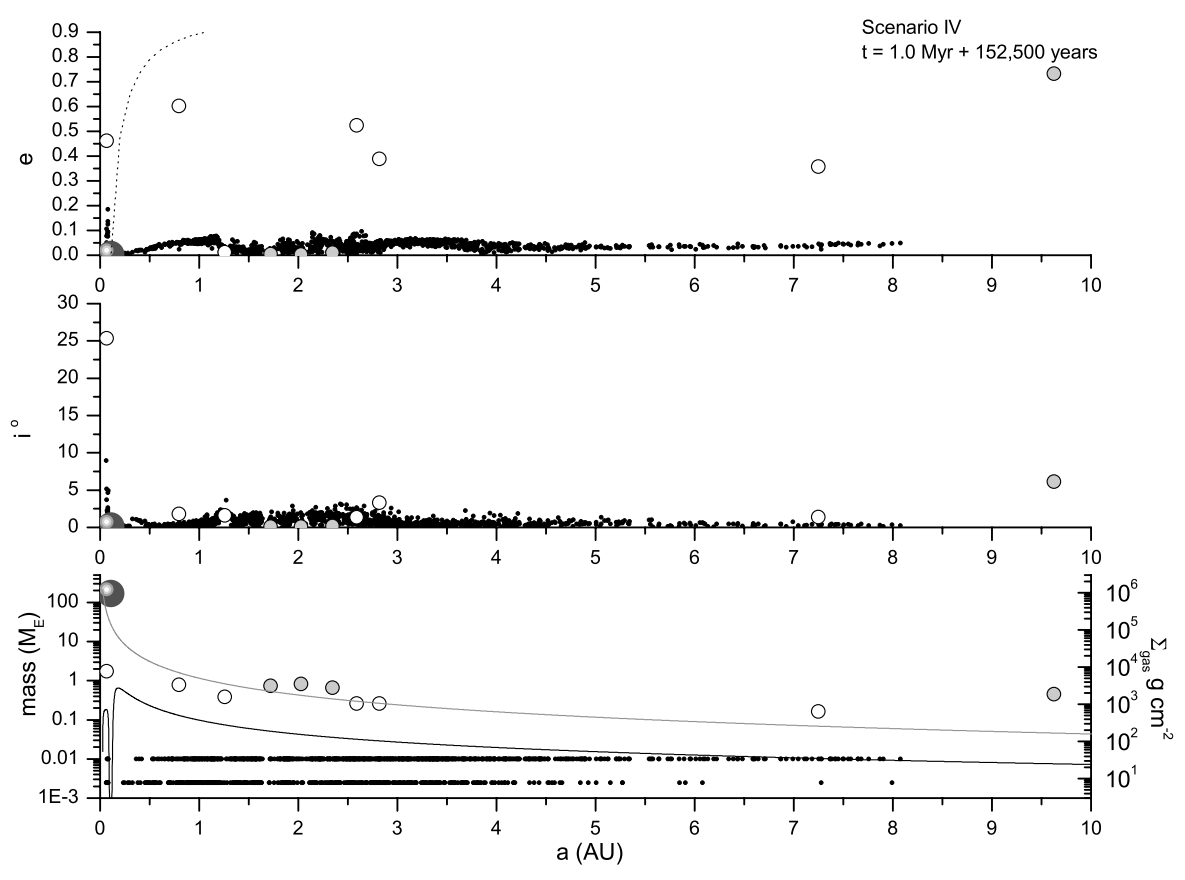

Fig. 6. The end point of scenario IV at 152500 years after the start of giant planet migration. The giant is at $0.1 \mathrm{AU}$. About half of the original solids disk mass survives in external orbits and seven protoplanets are found between 0.7-3 AU. A hot-Earth remains interior to the giant planet in an eccentric and inclined orbit.

Table 3. Fate of the disk mass at the end of each run: results for scenarios without $(\nrightarrow)$ and with $(\rightarrow)$ type I migration.

\begin{tabular}{c|cc|cc|cc}
\hline \hline Scenario & $\mathrm{I} \rightarrow$ & $\mathrm{I} \rightarrow$ & $\mathrm{IV} \rightarrow$ & $\mathrm{IV} \rightarrow$ & $\mathrm{V} \rightarrow$ & $\mathrm{V} \rightarrow$ \\
\hline \hline Total initial solids $\left(M_{\oplus}\right)$ & 24.81 & 24.81 & 24.81 & 24.81 & 24.81 & 23.47 \\
\hline Total surviving solids $\left(M_{\oplus}\right)$ & $16.60(67 \%)$ & $18.70(75 \%)$ & $21.23(86 \%)$ & $15.13(61 \%)$ & $20.22(81 \%)$ & $18.23(78 \%)$ \\
\hline Interior surviving solids $\left(M_{\oplus}\right)$ & $0.88(4 \%)$ & $3.44(14 \%)$ & $0.84(3 \%)$ & $1.84(7 \%)$ & $0.31(1 \%)$ & $6.23(27 \%)$ \\
$N, f_{\text {proto }}$ & 0,0 & $1,0.99$ & 0,0 & $1,0.96$ & 0,0 & 1,1 \\
\hline Exterior surviving solids $\left(M_{\oplus}\right)$ & $15.72(63 \%)$ & $15.27(62 \%)$ & $20.39(82 \%)$ & $13.29(54 \%)$ & $19.90(80 \%)$ & $12.00(51 \%)$ \\
$N, f_{\text {proto }}$ & $39,0.27$ & $34,0.22$ & $31,0.63$ & $9,0.34$ & $23,0.66$ & $7,0.24$ \\
\hline Accreted by star $\left(M_{\oplus}\right)$ & $0.01(0.04 \%)$ & $0.0(0 \%)$ & $0.0(0 \%)$ & $0.0(0 \%)$ & $0.0(0 \%)$ & $0.01(0.04 \%)$ \\
\hline Accreted by giant $\left(M_{\oplus}\right)$ & $8.20(33 \%)$ & $6.01(24 \%)$ & $3.41(14 \%)$ & $9.68(39 \%)$ & $4.59(19 \%)$ & $5.23(22 \%)$ \\
\hline Ejected $\left(M_{\oplus}\right)$ & $0.00(0 \%)$ & $0.1(0.4 \%)$ & $0.17(1 \%)$ & $0.0(0 \%)$ & $0.0(0 \%)$ & $0.0(0 \%)$ \\
\hline
\end{tabular}

compacted into an ever shrinking volume, underwent a series of violent mutual impacts followed by eventual accretion by the giant planet. A close encounter with the last of these hot-Earths to hit the giant was responsible for the high inclination of the orbit of the one survivor which, at $i=25.3^{\circ}$, is too low for the Kozai mechanism to play a role in subsequent orbital evolution. Despite these losses however, about half the mass of the original solids disk survives in the scattered external disk and most of this at radial distances $0.7<r<3$ AU (see the magnified illustration in Fig. 7). Seven substantial protoplanets ranging in mass from $0.26-0.82 M_{\oplus}$ now occupy this region along with a large quantity of yet to be accreted planetesimals. Type I eccentricity damping has already circularized the orbits of four of the protoplanets which might be expected to undergo some degree of renewed inward type I migration before complete gas dispersal followed by a final phase of planetesimal clear-up and giant impacts. Allowing for this, the configuration shown in Fig. 7 suggests a good chance that the final planetary arrangement here could include a planet in the system's habitable zone and we return to this issue in Sect. 3.3.

\subsection{Comparison with simulations not including type I migration}

Data showing the fate of the solids disk mass at the end of the three scenarios presented here that include type I migration are given in Table 3 in the columns headed with a $\rightarrow$ symbol and are compared against their counterparts from Fogg \& Nelson (2007) in the columns headed with a $\rightarrow$ symbol. Each column gives the total mass of solid material in the disk at the start of giant planet migration; the mass and the percentage of the initial disk surviving at the end of the migration; and the mass surviving interior and exterior to the giant planet's final orbit at $0.1 \mathrm{AU}$, including the number of remaining protoplanets and the protoplanetary mass fraction in each respective partition. The bottom three rows give the data for the mass loss channels which are accretion by the central star, accretion by the giant planet and ejection into a hyperbolic orbit.

These data show that a large fraction of the disk mass survives the passage of the giant planet in each case: inclusion of type I migration at the nominal rate does not result in the inner system being cleared of planet-forming material. In the two versions of scenario I slightly more mass remains with type I migration operating than without. However, this is largely due to the presence of a $3.42 M_{\oplus}$ hot-Earth inside 0.1 AU captured in the 3:2 resonance with the giant planet with an eccentricity of 0.29 . Generally the data for scenarios $\mathrm{I} \rightarrow$ and $\mathrm{I} \rightarrow$ are similar, suggesting that type I migration only has a small influence on the outcome of giant planet migration through a young inner system disk where protoplanetary masses remain small. In our more mature scenarios IV and V we do find less solids mass surviving when type I migration is included, but this loss is not 


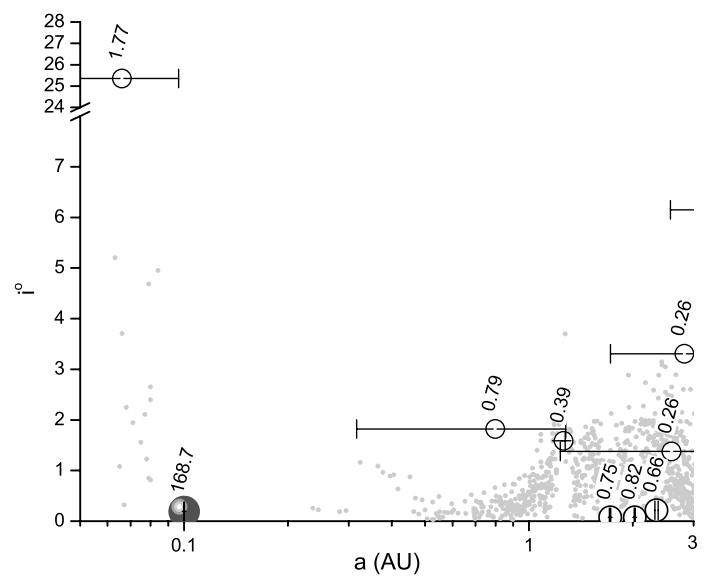

Fig. 7. Scenario IV interior to $3 \mathrm{AU}$ at the point where the giant planet reaches $0.1 \mathrm{AU}$. Inclination is plotted vs. semi-major axis. Super planetesimals are the grey dots and protoplanets the open circles. Planets also have marked their masses in $M_{\oplus}$ and their radial excursions due to orbital eccentricity.

catastrophic: $61 \%$ of the solids disk remains at the end of scenario IV (see Figs. 6 and 7) and 78\% remains at the end of scenario V. Almost exclusively, the mass that is lost is accreted by the giant planet. The increase in efficiency of this loss channel results from the fact that, due to previous inward type I migration, protoplanetary encounters with the giant planet tend to occur at smaller orbital radii where the probability of accretion versus scattering is relatively enhanced. In senario $\mathrm{V}$ this additional mass loss is minor as much of the inner system material had by this late time accreted into substantial protoplanets which were able to migrate ahead of the giant inside $0.1 \mathrm{AU}$ : a situation that can be seen developing in Fig. 2. These eventually coaccreted to form a $6.23 M_{\oplus}$ hot-Earth that was captured into the $2: 1$ resonance with the giant planet near the end of the simulation exciting its orbit to an eccentricity of 0.41 . Future accretion of this body by the giant planet is a possibility and we examine the issue of the long-term survival of our hot-Earth planets in Sect. 3.4.

Across the board in Table 3, the consistent trends that emerge when type I migration is added to our model are:

1. Shepherding is modestly enhanced. This can be seen from the larger percentage of the original solids disk mass that is found in the interior surviving solids fraction, which includes in each case a single hot-Earth type planet with an eccentric orbit captured at a first order resonance with the giant planet. Such objects were seen to form, but not to survive, in our analogous runs in Fogg \& Nelson (2007): their typical fate being accretion by the giant planet. The hot-Earths generated here have narrowly escaped this fate themselves and their long-term survival would depend on the cessation of any further inward migration of the giant and the effectiveness of any continuing type I eccentricity damping exerted by the tenuous gas (reduced by over two orders of magnitude from a $\Sigma_{\mathrm{g}} \propto r^{-1.5}$ model) remaining in the planet's vicinity. We do not recover the well-damped hot-Neptune or hotEarth type planets generated in Fogg \& Nelson (2005) where we assumed a steady state gas disk with a fixed $\Sigma_{\mathrm{g}} \propto r^{-1.5}$ surface density profile. In this case, the persistently high gas densities caused more rapid orbital decay of planetesimals, strong dynamical friction and high rates of collisional damping which were sufficient to drag several protoplanets into

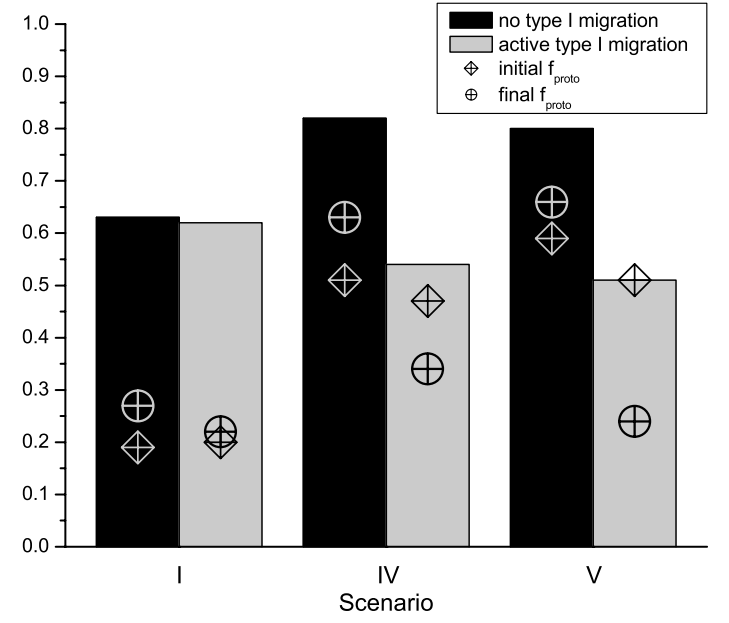

Fig. 8. Mass fractions of scattered disks generated in the present work compared to those of analogous scenarios not including type I migration and before and after protoplanet mass fractions.

low orbits where a rapid final phase of accretion was often sufficient to free the remaining body from resonant locking with the giant planet. Here, our evolving and decaying gas disk weakens both dynamical friction and type I eccentricity damping; whilst type I orbital decay facilitates inward movement of protoplanets, at late times it also brings about a separation of protoplanets from the bulk of planetesimals, further reducing the influence of dynamical friction on the innermost bodies. The overall dissipation in our present model therefore only results in a maximum of $27 \%$ of the original solids disk partitioned into the inner remnant.

2. Scattering is modestly reduced and strongly selective. Our simulations that include type I migration produce less massive scattered disks, but not dramatically so. A stronger effect is that the likelihood of external scattering is now biassed in favor of planetesimals, generating immature exterior solids disks with fewer protoplanets. This can be seen in Fig. 8 where the black bars show the mass of the scattered disk resulting from runs with no type I migration and the grey bars show those resulting from the present work. The superimposed lozenge symbols show the disk protoplanet mass fraction $\left(f_{\text {proto }}\right)$ at the start of each scenario and the circle symbols give the value of $f_{\text {proto }}$ at the end. In late scenarios the addition of type I migration to our model has resulted in the reduction of the mass of the outer disk remnant by $\sim 30 \%$ which still leaves $\sim 50 \%$ of the mass of the original ending up in the scattered disk. These disks however are less mature in the sense that they contain a greater fraction of small bodies. In Fogg \& Nelson (2007) it was noted that one effect of giant planet migration through a solids disk was to increase $f_{\text {proto }}$ in the external remnant by selectively scattering protoplanets. Figure 8 shows that in late scenarios, this trend is reversed here as type I migration, especially at late times, causes protoplanets to be preferentially shepherded: encounters with the giant tend to occur closer in so that fewer of them escape into the external remnant allowing the scattered planetesimal population to dominate. The external disks generated here however, though of reduced mass, should still be capable of supporting future planet formation. The protoplanets they contain can be of substantial mass and are typically emplaced at smaller semi-major axes and the more abundant planetesimal population and residual type I 


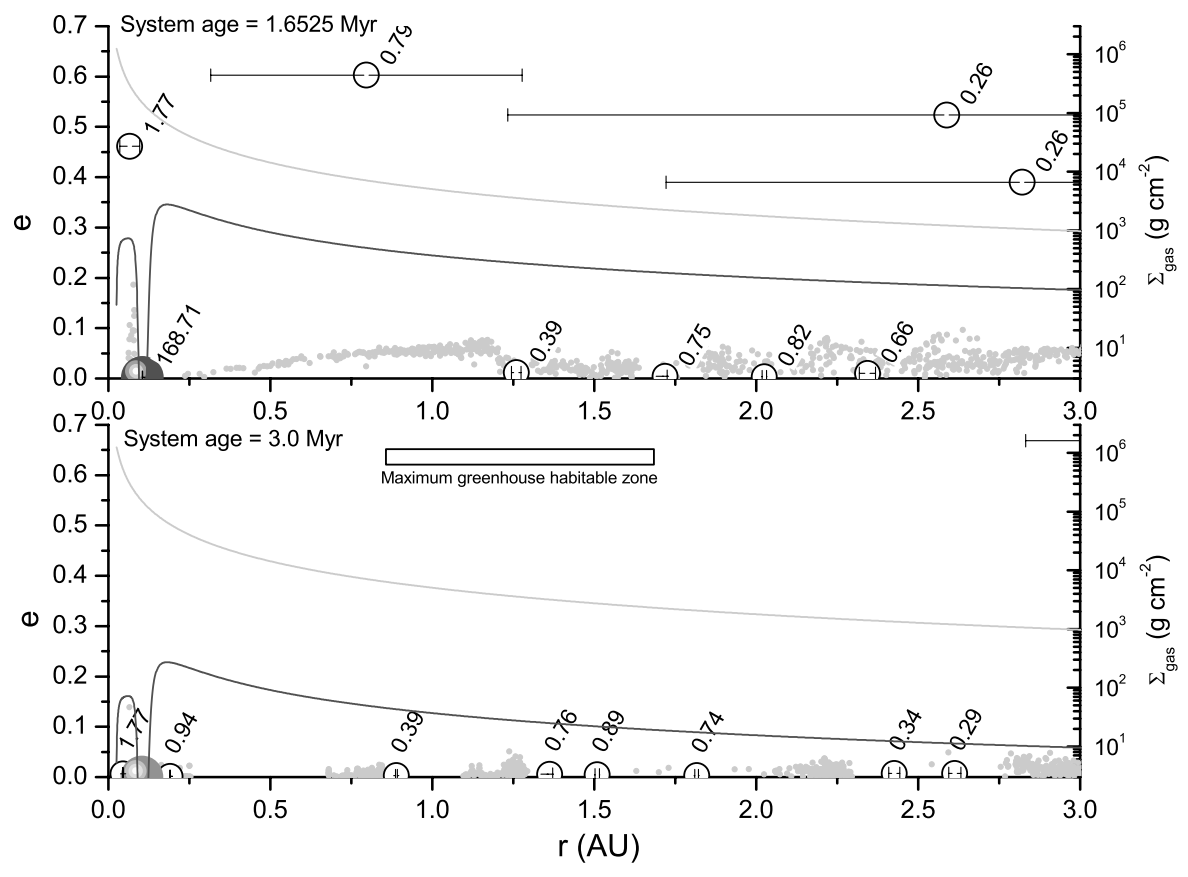

Fig. 9. Details of the inner $3 \mathrm{AU}$ at the end of scenario IV (top panel) and $1.3475 \mathrm{Myr}$ later at the point of gas dispersal. Eccentricity is plotted against semi-major axis with orbital excursions due to eccentricity illustrated with error bars. Planetary masses are labeled in units of $M_{\oplus} . \Sigma_{\mathrm{g}}$ is read from the right hand axes: the upper curve being the profile at $t=0$ (system age $=0.5 \mathrm{Myr}$ ) and the lower curve being the present profile. The extent of the maximum greenhouse habitable zone (0.8-1.7 AU) is shown by the horizontal bar. migration forces provide for stronger damping of protoplanets after scattering has occurred.

\subsection{Post-migration terrestrial planet formation}

To model the completion of planet formation in our scattered disks would require simulating a further $\sim 100 \mathrm{Myr}$ of accretion and is beyond the scope of this paper. Shorter range integrations however are still of considerable interest as type I migration forces only decline to zero when all the remaining local gas has gone. In this section, we continue the evolution of scenario IV to a system age of $3 \mathrm{Myr}$, requiring an additional $1.3475 \mathrm{Myr}$ of simulated run time, at which point we assume all residual gas is rapidly lost to photoevaporation. Over this period the mass of the gas disk falls from $\sim 5.5 M_{\mathrm{J}}$ to $\sim 0.55 M_{\mathrm{J}}$. In order to evade the severe limitations of our viscous disk algorithm time-step, which adapts to a tiny value with the giant planet at $\sim 0.1 \mathrm{AU}$, we turn off the type II migration forces effecting the hot-Jupiter and replace our viscous disk with an exponentially declining disk that maintains the previous surface density profile and declines with a mass e-folding time of 581514 years. We evolve the system henceforth with a 2 day $N$-body time-step. This set-up assumes an unspecified migration halting mechanism on the part of the hot-Jupiter that is not fully consistent with our model and we comment on the consequences of this in Sect. 4.

Details of the evolution of the inner $3 \mathrm{AU}$ of the system during this terminal phase of gas depletion are shown in Fig. 9 where the top panel shows the state of play at the end of scenario IV and the bottom panel shows it at $1.3475 \mathrm{Myr}$ later at a system age of 3 Myr. During this period we find that dynamical friction and residual type I migration forces rapidly damp and circularize the orbits of protoplanets with high eccentricities. Gas drag is also sufficient to maintain the eccentricities of planetesimals at a low value. The four well damped protoplanets between 1.25-2.5 AU in the upper panel of Fig. 9 grow by a few percent from the surrounding planetesimal field and migrate inward an additional $\sim 0.6 \mathrm{AU}$. By the time we remove the remaining gas in the bottom panel, two more planets have moved into the maximum greenhouse habitable zone (Kasting et al. 1993)

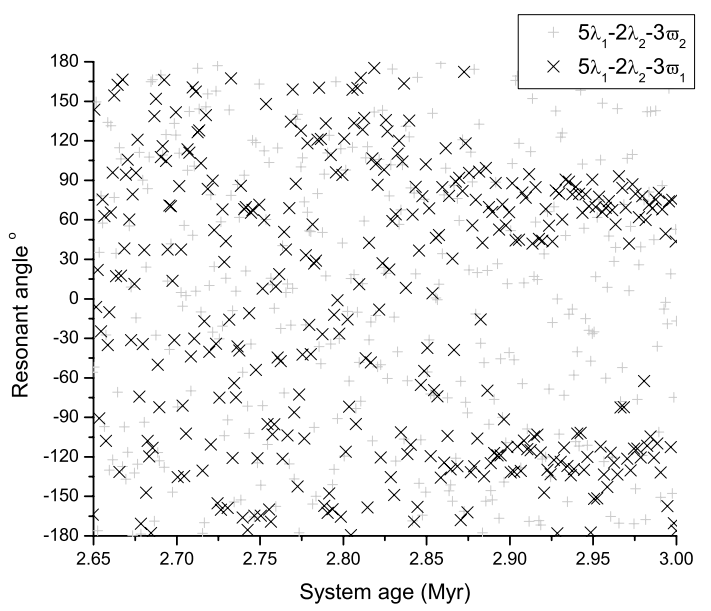

Fig. 10. Resonant angles for the 5:2 mean motion resonance between the giant planet and the innermost external protoplanet. Resonant capture is indicated $\sim 125000$ years before the stage shown in the bottom panel of Fig. 9.

giving a total of three planets sited between $0.8-1.7 \mathrm{AU}$. The migration of these four protoplanets shows some of the convoying behavior described in McNeil et al. (2005): convergent migration can cause protoplanets to bunch together and lock into a stable arrangement of mean motion resonances whereupon the pattern then migrates inward at some average drift rate. In the bottom panel of Fig. 9 the $0.76 M_{\oplus}$ planet and its $0.89 M_{\oplus}$ neighbour are caught at their 7:6 resonance and the outermost of this pair is close to the $4: 3$ resonance with its outer $0.74 M_{\oplus}$ neighbour. The innermost external protoplanet, having only recently been scattered, starts with $m_{\text {proto }} \simeq 0.78 M_{\oplus}, a \simeq 0.8$ AU and $e \simeq 0.6$ but damps rapidly and migrates inward, growing to $0.94 M_{\oplus}$ and eventually halting at $a=0.19 \mathrm{AU}$ at the $2: 5$ resonance with the hot-Jupiter (see Fig. 10). This behavior is an example of the tendency, when convergent migration is in play, for giant planets to collect smaller bodies at external mean motion resonances (Thommes 2005). 


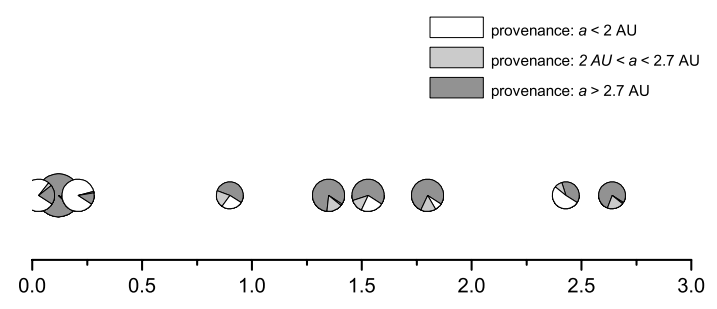

Fig. 11. Material composition of protoplanets in the extended scenario IV at the stage shown in the bottom panel of Fig. 9.

Our model also tracks the volatile composition of protoplanets, recognizing three crude material provenances: material originating at $a<2 \mathrm{AU}$ is assumed to be dry rock; from between $2 \mathrm{AU}<a<2.7 \mathrm{AU}$ its water content is assumed to be similar to that of chondritic asteroids; and from beyond $a>2.7$ AU it is assumed to be water-rich trans-snowline material. Previous models have demonstrated how a migrating giant planet drives volatilerich material into its inner system, boosting the potential water content of any planets that form there (Raymond et al. 2006; Fogg \& Nelson 2007; Mandell et al. 2007). This trend is accentuated when type I migration forces are included as volatilerich protoplanets can now migrate directly inward. In Fig. 11 we show the composition of the protoplanets in the extended scenario IV at a system age of 3 Myr. All the bodies have a substantial endowment of outer solids disk material especially those at $a>0.8 \mathrm{AU}$. The largest three of these planets, the resonant trio situated between 1.3-1.9 AU, all originated from beyond the snowline at $a>2.7 \mathrm{AU}$ and accreted copious volatile-rich material during their inward journey. Ocean worlds are a probable outcome of this kind of evolution (Kuchner 2003; Léger et al. 2004).

We have evolved this system, in the absence of gas, for a further $3 \mathrm{Myr}$ to a system age of $6 \mathrm{Myr}$ and two configurations over this period are shown in Fig. 12. The cessation of type I migration forces causes a shift to a more oligarchic style of growth (Kokubo \& Ida 1998). Orbital repulsion breaks up the resonant convoy showing in Fig. 9 and the entire protoplanet chain dynamically expands. The cessation of gas drag results in planetesimal orbits becoming much more excited in the process of exerting dynamical friction. Although this reduces protoplanetary accretion cross-sections, protoplanets no longer shepherd gaps in the solids disk and continuing growth is in progress.

The final state of the system shown in the bottom panel of Fig. 12 cannot be predicted in detail. We speculate that planetesimals will eventually be accreted or cleared, with some small fraction of them possibly surviving in the prominent gap in the planetary distribution at $\sim 0.5 \mathrm{AU}$. Future mergers of protoplanets via giant impacts are less certain as they all lie in nearcircular and well-spaced orbits: the closest being the two bodies between 1-1.5 AU which are nonetheless separated by $\sim 19 \mathrm{mu}-$ tual Hill radii. We expect therefore that the final configuration of the Scenario IV system will contain either one or two Earthmass planets in its habitable zone and longer range integrations of this system are underway.

\subsection{Hot-Earth survival}

Having used a 2 day time-step, the additional simulation represented by Fig. 12 does not adequately resolve the orbital evolution of the hot-Earth planet at 0.062 AU. Single hot-Earths, captured at interior first order mean motion resonances resulted from all our scenarios presented here, but their eccentric orbits with apastra close to the radial distance of the giant planet raise questions over long term survival. We have examined this issue by running the orbits of our hot-Earth candidates for a further 10000 years $\left(\sim 10^{6}\right.$ orbits at $\left.0.05 \mathrm{AU}\right)$, with a half day time-step, in the absence of gas and including the giant planet and any remaining debris interior to $0.1 \mathrm{AU}$. This assumes the existence of an interior gaseous cavity which is not fully consistent with our scenario end points and we comment on this further in Sect. 4.

The orbital evolution resulting from these extended runs is illustrated in Fig. 13 and their final configuration is shown in Fig. 14. All the interior planets remain. The least convincing as a longer-term survivor is the $3.43 M_{\oplus}$ hot-Earth present in Scenario I: it accreted very little additional debris (which was accreted by the giant instead) and continues to orbit at the 3:2 resonance with an eccentricity of $\simeq 0.3$, bringing it very close to crossing the giant's orbit at apastron. Close encounters have so far been avoided by the orbital phasing resulting from the resonance, but we have not investigated how long this state could persist. In the case of Scenario IV, we start with a $1.77 M_{\oplus}$ hot-Earth at the $2: 1$ resonance in an excited orbit with $e=0.46$ and $i=25^{\circ}$, but this planet succeeds in accreting an extra $0.03 M_{\oplus}$ of debris, cleaning up all of it but the one remaining super-planetesimal showing in Fig. 14, with the result that its eccentricity is damped to $e \simeq 0.2$ by the end of the run period. Its inclination is not damped and one accretion event increases it slightly to $i \simeq 30^{\circ}$ but the hot-Earth and the hot-Jupiter are now well separated and long-term survival looks promising. In scenario $\mathrm{V}$ there was no debris for the hot Earth to clean up and Fig. 13 shows that its orbit remains unchanged over 10000 years. This $6.23 M_{\oplus}$ planet remains close to the $2: 1$ resonance in a very low inclination orbit with $e \simeq 0.41$ which at no point comes dangerously close to the orbit of the giant planet. This hot-Earth is also a possible longterm survivor, especially as stellar tidal forces would be expected to gradually circularize its orbit over much longer timescales.

\section{Discussion}

Any simulation of planet formation is restricted by its assumptions and incomplete input physics. Below, we discuss three areas where future improvements are possible as well as the caveats they might hold for our present results.

(i) Migration halting mechanisms. In all our simulations we have halted the migration of our giant planet at $0.1 \mathrm{AU}$ where we assume that whatever mechanism is responsible for stranding hot-Jupiters in their final orbits comes into play. This mechanism is unknown and proposed explanations include the presence of a central magnetospheric cavity in the gas which could decouple an intruding giant planet from the disk (Lin et al. 1996); another is that of fortuitous gas disk dispersal where the eventual loss of the gas disk strands those migrating giant planets that still remain at arbitrary orbital radii (Trilling et al. 1998). The former could apply to any of our scenarios, early or late, whilst the latter is more appropriate to late scenarios when most of the gas has already dispersed. Neither of these mechanisms are fully consistent with our present model. If we were to run it further, we would expect the giant planet to come to rest somewhere between $0.02-0.1$ AU but we have yet to model any of the physics of a magnetospheric cavity and time-step limitations that stem from our viscous disk algorithm make it impractical to simulate further type II migration interior to $0.1 \mathrm{AU}$. The only difference that stopping the hot-Jupiter closer to the star would make to our results is that more compact systems would be less likely to host interior hot-Earth or hot-Neptune type planets. Further resonant shepherding of such objects inward of $0.1 \mathrm{AU}$ runs the 


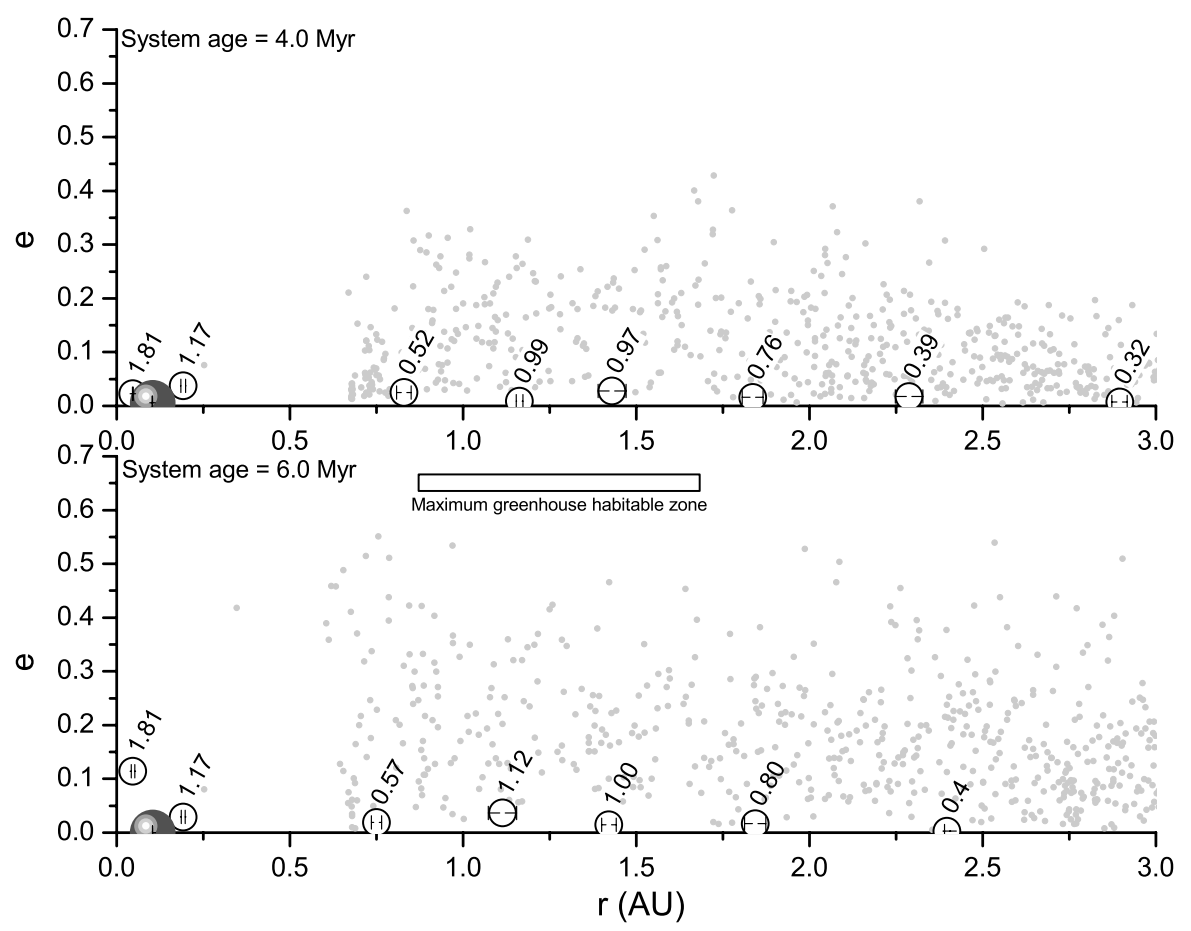

Fig. 12. Details of the inner 3 AU of the Scenario IV system after $3 \mathrm{Myr}$ of gas-free dynamics. The top and bottom panels show configurations at system ages of 4 and $6 \mathrm{Myr}$ respectively.

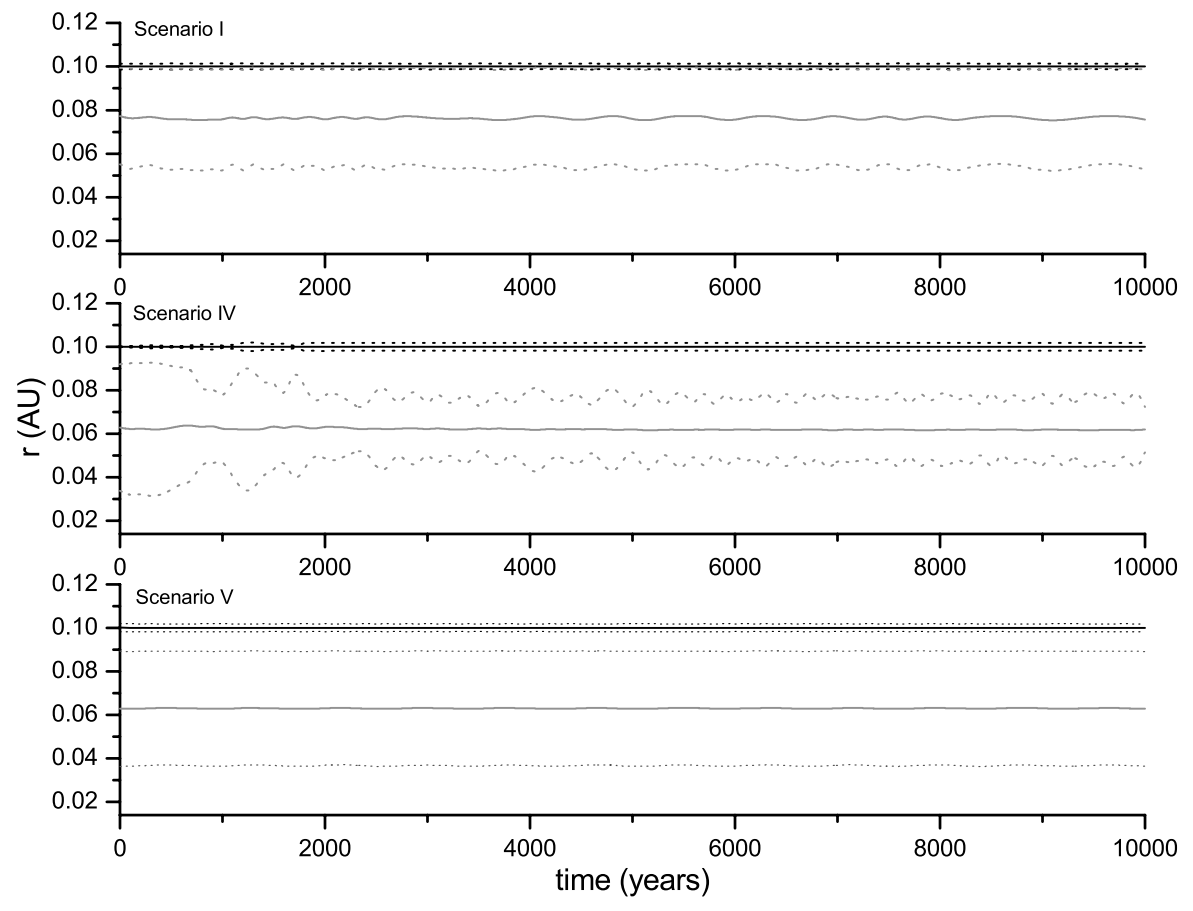

Fig. 13. Further evolution of the orbits of the interior planets resulting from the three scenarios. Solid lines are semi-major axes, dotted lines are periastra and apastra. Black represents the giant planet and grey represents the hot-Earth. risk of driving them into the central star (Fogg \& Nelson 2005). Our results for scattered disk characteristics and their prospects of renewed terrestrial planet formation would be unaffected by the final fate of the hot-Jupiter. If fortuitous disk dispersal is assumed to be the halting mechanism, then this could be simulated in our model by including a photoevaporation algorithm which removes gas at some small and steady rate that accords with observations. This would result in a rapid dispersal of the of the remaining gas at late times (e.g. Clarke et al. 2001; Alexander et al. 2006) and a termination of type II migration. Work is ongoing to include this mechanism in our model and to conduct simulations where giant planets strand at a variety of distances in a fully self-consistent way. (ii) Supply of outer disk material. In our previous models the radial drift of protoplanets is due to dynamical stirring and a degree of coupling to the slow inward movement of planetesimals via dynamical friction. In these cases it was reasonable to assume that our giant planet formed at $5 \mathrm{AU}$ and that any supply of extra material over our run times into the inner disk from beyond that distance would be insignificant. However the inclusion of type I migration makes the heaviest and most evolved objects more inwardly mobile. As shown in Fig. 2, our late matured disks are largely cleared of protoplanets outward of $\sim 2 \mathrm{AU}$, making one wonder if this region could have been repopulated in the interim by objects which migrated from beyond 4 AU. This seems plausible as the giant planet's core must also have 


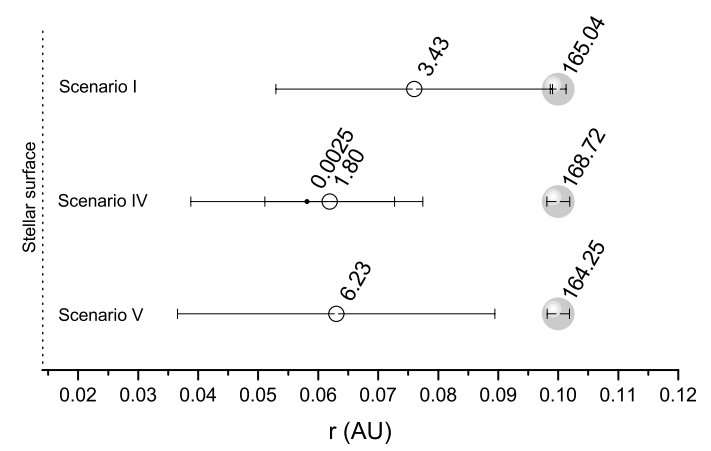

Fig. 14. Hot-Earth-hot-Jupiter systems resulting from an additional 10000 years of simulation. Error bars show the radial distance between periastron and apastron. Numeric labels give masses in $M_{\oplus}$.

migrated so that we can no longer propose consistently that it accreted its core at $5 \mathrm{AU}$. We have not yet attempted to model the extent of any such addition of external mass to our solids disk but it is clear from our results that any additional protoplanets encountered by the giant between 2-4 AU are more likely to be scattered than accreted. Thus, any late supply of large bodies is likely to contribute to the population of the scattered disk boosting both its mass, maturity $\left(f_{\text {proto }}\right)$, and volatile content.

(iii) Planetesimal size evolution. We have assumed a uniform population size of $10 \mathrm{~km}$ planetesimals which results in an identical effect of gas drag on any planetesimal at a particular orbital distance. In reality, but more challenging to adapt to a $N+N^{\prime}$-type simulation, we would expect an evolving planetesimal size spectrum and a much more varied response of small objects to gas drag. In areas of the solids disk where planetesimals are strongly stirred, such as between the 2:1 and 4:3 resonances with the giant planet, high energy mutual collisions might reduce planetesimals down to smaller chunks of rubble. This rubble would be subject to stronger dissipation from the gas resulting in it being shepherded inward of the giant more efficiently. In Fogg \& Nelson (2007) we suggested that much of this material should still end up in the scattered disk as it would be expected to accrete very efficiently onto inner protoplanets (see Chambers 2006b) that would be scattered later. This argument is weaker when type I migration is included. Supplying inner planets with an enhanced feedstock would not only boost their growth rates, but also their inward drift. We have shown in Sect. 3.2 how the influence of type I migration results in fewer protoplanets surviving in external orbits so boosting inward migration rates by enhancing mass growth would accentuate this trend. However, as accretion rates depend as $\dot{m}_{\text {proto }} \propto \Sigma \Omega$ we would expect that the bulk of this additional growth would take place at late times when the giant planet has compacted the shepherded fraction within a small orbital radius. It seems unlikely therefore that the additional dissipation resulting from planetesimal fragmentation would prevent entirely the scattering of some large bodies into the external disk.

(iv) Initial nebular surface density profile. In common with many other studies of terrestrial planet formation, our models adopt an initial condition of a MMSN-type protoplanetary disk with an $r^{-1.5}$ surface density profile (Weidenschilling 1977; Hayashi 1981). Other models however predict that a shallower profile $\left(\Sigma \sim r^{-1}\right)$ may be more realistic (e.g. Davis 2005; Andrews \& Williams 2007). We have not yet explored the effect of running our present model with a shallower initial profile but expect that it would not drastically change our results. Previous models that have explored this issue (Chambers \& Cassen 2002;
Raymond et al. 2005b; McNeil et al. 2005, e.g.) find that the architectures of their resulting terrestrial planetary systems depend only weakly on the slope of the initial surface density set up with shallower gradients tending to result in more substantial planets forming further from the central star. If a giant planet were to migrate through such a disk, we expect that more mass would be encountered earlier in the migration, resulting in a more massive scattered disk and hence even more favorable prospects for external terrestrial planet formation.

\section{Conclusions}

Previous models of a giant planet migrating through an inner system protoplanetary disk have all predicted that the process generates a scattered external disk massive enough to support renewed planet formation (Fogg \& Nelson 2005, 2006; Raymond et al. 2006; Fogg \& Nelson 2007; Mandell et al. 2007). None of these works included the possible action of type I migration forces exerted by the gas which would cause heavier bodies to drift progressively closer to the central star. Since this process would clearly act to counter scattering we have questioned if its operation could invalidate previous conclusions concerning subsequent terrestrial planet formation. Having updated our model to include type I migration and having re-run three scenarios from Fogg \& Nelson (2007), we conclude that the extra dissipation generated is still not sufficient to prevent the buildup of a scattered disk $\sim 50 \%$ the mass of the original. Although these scattered disks are of lower mass, they are generally less dispersed and better damped, supporting a more rapid renewal of planetary growth. As shown in our extended run, whilst the gas remains, residual type I forces can act on scattered protoplanets to draw them back into the $\sim 1 \mathrm{AU}$ region and the habitable zone. Our model also generates planets of several Earth masses in potentially stable orbits interior to the final orbit of the giant planet and is thus supportive of the proposals of Fogg \& Nelson (2005) and Zhou et al. (2005) that hot-Earths and hot-Neptunes found interior to hot-Jupiters may have originated from material shepherded inward by a migrating giant planet.

Hot-Jupiter systems therefore, far from being devoid of other planetary bodies within their warmer regions, may be worthwhile targets for future investigation.

Acknowledgements. We thank the referee, Sean Raymond, whose comments led to improvements in this paper.

\section{References}

Alexander, R. D., Clarke, C. J., \& Pringle, J. E. 2006, MNRAS, 369, 229 Andrews, S. M., \& Williams, J. P. 2007, ApJ, 659, 705

Armitage, P. J. 2003, ApJ, 582, L47

Bertout, C. 1989, ARA\&A, 27, 351

Boss, A. P. 2000, ApJ, 536, L101

Butler, P., Wright, J. T., Marcy, G. W., et al. 2006, ApJ, 646, 505

Chambers, J. E. 1999, MNRAS, 304, 793

Chambers, J. E. 2001, Icarus, 152, 205

Chambers, J. E. 2006a, ApJ, 652, L133

Chambers, J. E. 2006b, Icarus, 180, 496

Chambers, J. E., \& Cassen, P. 2002, M\&PS, 37, 1523

Clarke, C. J., Gendrin, A., \& Sotomayor, M. 2001, MNRAS, 328, 485

Cresswell, P., \& Nelson, R. P. 2006, A\&A, 450, 833

Daisaka, J. K., Tanaka, H., \& Ida, S. 2006, Icarus, 185, 492

Davis, S. S. 2005, ApJ, 627, L153

Fogg, M. J., \& Nelson, R. P. 2005, A\&A, 441, 791

Fogg, M. J., \& Nelson, R. P. 2006, Int. J. Astrobiol., 5, 199

Fogg, M. J., \& Nelson, R. P. 2007, A\&A, 461, 1195

Gomes, R., Levison, H. F., Tsiganis, K., \& Morbidelli, A. 2005, Nature, 435, 466

Haisch, K. E., Lada, E. A., \& Lada, C. J. 2001, ApJ, 553, L153

Halliday, A. N. 2004, Nature, 427, 505 
Hayashi, C. 1981, Prog. Theor. Phys. Suppl., 70, 35

Jones, B. W., Underwood, D. R., \& Sleep, P. N. 2005, ApJ, 622, 1091

Kasting, J. F., Whitmire, D. P., \& Reynolds, R. T. 1993, Icarus, 101, 108

Kleine, T., Münker, C., Mezger, K., \& Palme, H. 2002, Nature, 418, 952

Kokubo, E., \& Ida, S. 1998, Icarus, 131, 171

Kokubo, E., \& Ida, S. 2000, Icarus, 143, 15

Kuchner, M. J. 2003, ApJ, 596, L105

Laughlin, G., Steinacker, A., \& Adams, F. C. 2004, ApJ, 608, 489

Léger, A., Selsis, F., Sotin, C., et al. 2004, Icarus, 169, 499

Lin, D. N. C., \& Papaloizou, J. C. B. 1986, ApJ, 309, 846

Lin, D. N. C., Bodenheimer, P., \& Richardson, D. C. 1996, Nature, 380, 606

Lineweaver, C. H. 2001, Icarus, 151, 307

Lineweaver, C. H., Fenner, Y., \& Gibson, B. K. 2004, Science, 303, 59

Mandell, A. M., Raymond, S. N., \& Sigurdsson, S. 2007, ApJ, 660, 823

Masset, F. S., Morbidelli, A., Crida, A., \& Ferreira, J. 2006, ApJ, 642, 478

McNeil, D., Duncan, M., \& Levison, H. F. 2005, AJ, 130, 2884

Morbidelli, A., Levison, H. F., Tsiganis, K., \& Gomes, R. 2005, Nature, 435, 462

Nagasawa, M., Lin, D. N. C., \& Thommes, E. 2005, ApJ, 635, 578

Nelson, R. P. 2005, A\&A, 443, 1067

Nelson, R. P., \& Papaloizou, J. C. B. 2004, MNRAS, 350, 849

Nelson, R. P., Papaloizou, J. C. B., Masset, F. S., \& Kley, W. 2000, MNRAS, 318,18

Nimmo, F., \& Kleine, T. 2007, Icarus, in press
O'Brien, D. P., Morbidelli, A., \& Levison, H. F. 2006, Icarus, 184, 39 Paardekooper, S.-J., \& Mellema, G. 2006, A\&A, 459, L17

Papaloizou, J. C. B., \& Larwood, J. D. 2000, MNRAS, 315, 823

Papaloizou, J. C. B., \& Nelson, R. P. 2005, A\&A, 433, 247

Pollack, J. B., Hubickyj, O., Bodenheimer, P., et al. 1996, Icarus, 124, 62

Raymond, S. N., Quinn, T., \& Lunine, J. I. 2005a, Icarus, 177, 256

Raymond, S. N., Quinn, T., \& Lunine, J. I. 2005b, ApJ, 632, 670

Raymond, S. N., Mandell, A. M., \& Sigurdsson, S. 2006, Science, 313, 1413

Takeuchi, T., Miyama, S. N., \& Lin, D. N. C. 1996, ApJ, 460, 832

Tanaka, H., \& Ward, W. R. 2004, ApJ, 602, 388

Tanaka, H., Takeuchi, T., \& Ward, W. R. 2002, ApJ, 565, 1257

Thommes, E. W. 2005, ApJ, 626, 1033

Thommes, E. W., \& Murray, N. 2006, ApJ, 644, 1214

Thommes, E. W., Duncan, M. J., \& Levison, H. F. 2003, Icarus, 161, 431

Thommes, E. W., Nilsson, L., \& Murray, N. 2007, ApJ, 656, L25

Trilling, D. E., Benz, W., Guillot, T., et al. 1998, ApJ, 500, 428

Tsiganis, K., Gomes, R., Morbidelli, A., \& Levison, H. F. 2005, Nature, 435, 459

Ward, P. D., \& Brownlee, D. 2000, Rare Earth: Why complex life is uncommon in the universe (New York: Copernicus Books)

Ward, W. R. 1997, Icarus, 126, 261

Weidenschilling, S. J. 1977, Ap\&SS, 51, 153

Zhou, J.-L., Aarseth, S. J., Lin, D. N. C., \& Nagasawa, M. 2005, ApJ, 631, L85 